\title{
3D (Three-Dimensional) Caco-2 Spheroids: Optimized in vitro Protocols to Favor Their Differentiation Process and to Analyze Their Cell Growth Behavior
}

\author{
Gabriella Rainaldi, Alessandra Boe and Sandra Gessani \\ Department of Hematology, Oncology and Molecular Medicine, Istituto Superiore di Sanità, Rome, Italy
}

\begin{abstract}
D (Three-dimensional) Caco-2 spheroids closely recapitulating in vivo physiological organization of intestinal epithelial cells, provide an excellent in vitro model system to study their pathophysiology and their response to stressful stimuli. The objective of this technical note is to provide optimized in vitro experimental protocols for culturing 3D Caco-2 spheroids and for analyzing their cell growth features. An optimized 3D Caco-2 spheroid culturing technique based on a new configuration of the culture medium is provided. A methodological approach to determine the distribution of the cell cycle phases in disaggregated Caco-2 spheroids by using cytofluorimetric analysis is also described. The optimized culturing protocol favors 3D Caco-2 spheroid differentiation process, as evaluated by the number of well-differentiated spheroids with a single hollow lumen. The cytofluorimetric analysis allows rapid collection of cell cycle phase data from high numbers of spheroid samples, thus, permitting to estimate their growth dynamics in a relatively short time. The optimized technical approaches described here can be applied in systematic manner to a variety of research activities utilizing 3D Caco-2 spheroids. Ease of use, time and economic saving advantages deriving from these protocols further highlight their potential.
\end{abstract}

Key words: 3D multicellular spheroids, 3D intestinal epithelial spheroids, 3D Caco-2 spheroid culture systems, optimized experimental protocols.

\section{Introduction}

For many years, 2D (two-dimensional) cell culture system (monolayers) has provided important information about cellular structure and function. However, in 2D monolayer cultures the 3D (three-dimensional) arrangement of real tissue and its interactions with the microenvironment are ignored. In this era of ever-expanding technological innovations in which the biological challenges to deal with are increasingly intricate, the use of advanced cellular systems is indispensable. Therefore, in an effort to design more sophisticated in vitro systems, that actually resemble the complex environment of native tissues, researchers have developed realistic in vitro $3 \mathrm{D}$

Corresponding author: Sandra Gessani and Gabriella Rainaldi, Department of Hematology, Oncology and Molecular Medicine, Istituto Superiore di Sanità, Viale Regina Elena 299, 00161 Rome, Italy. E-mail: sandra.gessani@iss.it; gabriella.rainaldi@iss.it. cell culture models [1-6]. In the last years, the potential of 3D cell models as pivotal tools in cellular physiology investigations has become more and more established. This awareness has intensified the use of 3D cell culture systems in various research fields, including drug discovery, pharmacological applications, cell physiology, cell death, gene and protein expressions, tissue engineering, and cancer research [7-9].

3D cell culture systems are an excellent in vitro model to gain information about intestinal epithelial pathophysiology $[10,11]$. Indeed, 3D intestinal epithelial spheroids quite precisely recreate the spatial organization of intestinal epithelial cells in vivo, thus, they offer the promise to realistically simulate intestinal epithelium responses to stressful stimuli [10, $12,13]$. In this context, 3D Caco-2 (human colorectal adenocarcinoma) spheroids are well-recognized 3D 
culture system for exploring intestinal functional and morphological features. In fact, when grown in a basement membrane matrix, Caco- 2 cells differentiate to 3D spheroids characterized by a single central lumen and expressing typical morphological traits of intestinal epithelium in vivo, such as polarization of cells, adherens and tight junctions, and microvilli [12, $14,15]$.

Aim of this technical note is to provide optimized in vitro experimental protocols for: i) culturing 3D Caco-2 spheroids, and ii) analyzing their cell growth characteristics. The first protocol describes an in vitro 3D Caco-2 spheroid culturing technique optimized to favor their differentiation process (experimental protocol 1). This method consists in significant variations of the 3D epithelial cell culture protocols used by other investigators to grow and differentiate 3D CaCo-2 spheroids [10, 12, 13, 15]. The principal modification is the use of an alternative configuration of the culture medium (Dulbecco's modified Eagle's medium-DMEM) to grow the $\mathrm{CaCo}-2$ spheroids, namely 5\% heat-inactivated FBS (Fetal Bovine Serum) and 2,75 g/L glucose final concentrations (hereafter, DMEM 2,75 g/l glucose-5\% FBS). The entire Caco-2 spheroid culture seeding protocol has been designed as a function of this medium formulation. The second protocol describes a technical approach to study 3D Caco-2 spheroid growth dynamics based on the distribution of the cell cycle phases $\left(\mathrm{G}_{0} / \mathrm{G}_{1}, \mathrm{~S}\right.$ and $\left.\mathrm{G}_{2} / \mathrm{M}\right)$ by using cytofluorimetric (FACS, fluorescence-activated cell sorting) analysis (experimental protocol 2). 3D Caco-2 spheroids are isolated from Matrigel cultures, disaggregated to obtain single cell suspensions and the cell number quantified, as previously described for other 3D epithelial spheroid types [16]. Our experimental protocol 2 consists in the specific FACS analysis of the single cell suspensions obtained from disaggregated Caco-2 spheroids to determine their cell cycle phase distribution.

\section{Methods}

In Table 1 are listed reagents and supplies used to grow and analyze 3D Caco-2 spheroids.

\subsection{Experimental Protocol 1: Guidelines for Optimized} 3 D Caco-2 Spheroid Culture Method (DMEM 2,75 g/l Glucose-5\% FBS)

In Table 2 are reported formulations of culture media used in experimental protocol 1.

Caco-2 cells (from the American Type Culture Collection, ATCC, Manassas, VA, USA) (passage 35-48) were grown as standard monolayer in tissue culture flasks in complete DMEM high glucose (Table 2 , medium A), at $37{ }^{\circ} \mathrm{C}$ in a $5 \% \mathrm{CO} 2$ atmosphere. 20

1 Matrigel was allowed to solidify at $37{ }^{\circ} \mathrm{C}$ for 30 minutes onto $12 \mathrm{~mm}$ sterile glass coverslips placed inside 24-well tissue culture plates. Caco-2 cell monolayers were detached from the substratum by using $0.02 \%$ EDTA and 0.25 trypsin solutions, both in PBS buffer. For culturing Caco-2 spheroids by using the DMEM formulation used by other investigators

Table 1 Reagents and supplies used to grow and analyze 3D Caco-2 spheroids.

\begin{tabular}{|l|}
\hline Reagents and supplies \\
\hline DMEM high glucose with 4,5 g/L Glucose (Lonza, N. BE12-614F) \\
\hline DMEM low glucose with 1,0 g/L Glucose (Gibco, N. 31600-083) \\
\hline FBS (Fetal Bovine Serum) Characterized (Hyclone, USA, N. SH30071.03) \\
\hline L-Glutamine $200 \mathrm{mM}$ (Lonza, N. BE17-605E) \\
\hline 10.000 U Penicillin/ml - 10.000 U Streptomicin/ml (Lonza, N. DE17-602E) \\
\hline MEM NEAA (Non-essential amino acids) 100X (Gibco, by Life Technologies, N. 11140-035) \\
\hline Matrigel @ Matrix Basement Membrane (Corning, MA, USA, 8.8 mg/ml, N. 356234) \\
\hline RNAsi (Sigma-Aldrich, N. R5125) \\
\hline Propidium Iodide (PI) (Sigma-Aldrich, N. P4170) \\
\hline Filcon $50 \quad$ m, Sterile, Syringe-Type (BD, N. 340602) \\
\hline
\end{tabular}


Table 2 Formulations of culture media used in experimental protocol 1.

\begin{tabular}{|c|c|}
\hline DMEM medium formulations & \\
\hline DMEM high Glucose (4,5 g/L Glucose) & \\
\hline Complete medium formulation (medium A): & Serum-free medium formulation (medium B): \\
\hline - 10\% heat-inactivated FBS & - None heat-inactivated FBS \\
\hline$-1 \% N E A A$ & $-1 \% N E A A$ \\
\hline - $1 \%$ Penicillin-Streptomycin & - $1 \%$ Penicillin-Streptomycin \\
\hline - $1 \%$ Glutamine & - $1 \%$ Glutamine \\
\hline DMEM low Glucose (1g/L Glucose) & \\
\hline Serum-free medium formulation (medium $C$ ). & \\
\hline - $1 \%$ Penicillin-Streptomycin & \\
\hline - $1 \%$ Glutamine & \\
\hline DMEM 2,75 g/L Glucose-5\% FBS & \\
\hline Medium composition (medium $D$ ): & \\
\hline - DMEM high glucose + DMEM low glucose & $1: 1(v / v)$ resulting in $2,75 \mathrm{~g} / \mathrm{L}$ Glucose \\
\hline - 5\% heat-inactivated FBS & \\
\hline - 0,5\% NEAA & \\
\hline - 1\% Penicillin-Streptomycin & \\
\hline - $1 \%$ Glutamine & \\
\hline
\end{tabular}

(DMEM 4,5 g/L glucose-10\% FBS),[10,12,13] Caco-2 cells (5000 cells/well) were resuspended in serum-free DMEM 4,5 g/L glucose (Table 2, medium B) mixed with $40 \%$ Matrigel (v/v), and the cell suspension was plated onto solidified Matrigel. After incubation at $37^{\circ} \mathrm{C}$ in a $5 \% \mathrm{CO} 2$ atmosphere, $2 \mathrm{ml}$ of DMEM 4,5 g/L glucose-10\% FBS (Table 2 medium A) were added to each well. In order to culture Caco-2 spheroids by using our optimized DMEM formulation (DMEM 2,75 g/l glucose-5\% FBS), Caco-2 cells (5000 cells/well) were resuspended in serum-free DMEM 4,5 g/L glucose (Table 2, medium B) and serum-free DMEM $1 \mathrm{~g} / \mathrm{L}$ glucose (Table 2, medium C) mixed together at ratio $1: 1(\mathrm{v} / \mathrm{v})$, and added with $40 \%$ Matrigel (v/v). Cell suspension was plated onto solidified Matrigel and, after incubation at $37^{\circ} \mathrm{C}$ in a $5 \%$ $\mathrm{CO} 2$ atmosphere, $2 \mathrm{ml}$ of DMEM 2,75 g/l glucose- $5 \%$ FBS (Table 2, medium D) were added to each well. All samples were incubated at $37^{\circ} \mathrm{C}$ in a $5 \% \mathrm{CO} 2$ atmosphere at least up to 9 days.

\subsection{D Caco-2 Spheroids Cell Growth Analyses}

2.2.1 Disaggregation Procedure of Caco-2 Spheroids and Cell Counting

To obtain single cell suspensions from 3D cultures,
Caco-2 spheroids embedded in the Matrigel were collected on ice and centrifuged at $1500 \mathrm{rpm}$ for 5 minutes at $4{ }^{\circ} \mathrm{C}$, as described for other types of epithelial spheroids [16]. Spheroids were disaggregated by gentle pipeting with $0.1 \%$ EDTA and $0.25 \%$ trypsin solutions both at $37{ }^{\circ} \mathrm{C}$. Trypsin action was stopped by adding medium containing $10 \%$ FBS. After centrifugation at $1500 \mathrm{rpm}$ for 5 minutes at $4{ }^{\circ} \mathrm{C}$, single Caco- 2 cell suspensions were resuspended in 1 $\mathrm{ml}$ of complete medium on ice, and counted using a Neubauer chamber.

2.2.2 Experimental Protocol 2: Guidelines for FACS Cell Cycle Analysis of Disaggregated CaCo-2 Spheroids

To determine the distribution of the cell cycle phases in disaggregated $\mathrm{CaCo}-2$ spheroids by using FACS analysis the following procedure was conducted.

Single cell suspensions, obtained with the disaggregation procedure described above, were fixed in ice-cold $70 \%$ ethanol in PBS for 10 minutes, centrifuged at $1500 \mathrm{rpm}$ for 5 minutes at $4{ }^{\circ} \mathrm{C}$, and the supernatants removed. Cell pellets were resuspended in PBS containing $125 \mathrm{~g} / \mathrm{ml}$ of RNAse and $50 \quad \mathrm{~g} / \mathrm{ml}$ of the DNA fluorescent probe PI and, after incubation at $37^{\circ} \mathrm{C}$ for 30 minutes, cell suspensions were filtered 
with Filcons filters $50 \mathrm{~m}$. Sample fluorescence was analyzed with a FACS Canto flow cytometer (Becton Dickinson, San Jose, CA, USA) using blue laser (488-nm, air cooled, 20-mW solid state). PI emission was measured using the BP 585/42 nm filter. PI was collected in a linear scale in order to clearly distinguish between the cell cycle phases. Acquisition was done at a low flow rate under 300 events/second. At least 10,000 single cells were collected per sample and analyzed using the FACS DIVA software (Becton Dickinson). To recognize the cell population the FS (forward scatter) and SC (side scatter) were measured. The gated population was plotted for PI area versus PI width to identify cell doublets and clumps which were gated out [17]. The PI histogram graph of this gated population showed the three distinct phases of a proliferating cell population: $\mathrm{G}_{0} / \mathrm{G}_{1}, \mathrm{~S}$ and $\mathrm{G}_{2} / \mathrm{M}$. The percentage of cells in each cell cycle phase was quantified by using markers set within analysis which was conducted using the FACS DIVA software (Becton Dickinson). The quality of the DNA histograms was estimated as previously described, ${ }^{[17]}$ i.e., by taking into consideration two pivotal parameters: i) the $\mathrm{CV}$ (coefficient of variation), that should be $\leq 5$; ii) the $G_{2} / G_{1}$ ratio, calculated on the Gaussian peak positions in the DNA content histogram, whose most common value is about 1.9. In our disaggregated Caco-2 spheroid samples, the $\mathrm{CV}$ obtained was about 5 , and the value for the $\mathrm{G}_{2} / \mathrm{G}_{1}$ ratio was about 1.9.

\subsection{Morphological Microscopic Analysis of Whole 3D} Caco-2 Spheroids

Matrigel embedded 3D Caco-2 spheroids were fixed with 1\% formaldehyde (Carlo Erba Reagents) in PBS for 15 minutes at RT (room temperature) and permeabilized with $0,5 \%$ Triton X-100 (Sigma-Aldrich) in PBS at RT. Actin filaments were labeled using Phalloidin-TRITC (Sigma-Aldrich) and nuclei were counterstained with Hoechst 33258 (Sigma-Aldrich), both for 30 minutes at RT. All samples were observed and photographed with a Zeiss Axiovert fluorescence microscope directly in the tissue culture plates.

\section{Results}

\subsection{Distinctive Morphological Characteristics of Fully Differentiated 3D Caco-2 Spheroids}

Fully differentiated 3D Caco-2 spheroids are characterized by distinct morphological features, namely, a single layer of cells which surrounds a central hollow lumen, and a ring of cytoskeletal actin filaments in the luminal side of these cells $[12,15]$. Consequently, independently of the experimental culture conditions used, fully differentiated Caco-2 spheroids have necessarily to comply with these distinctive ultra structural parameters (Fig. 1). Furthermore, as Caco-2 spheroids have a 3D spherical architecture, to provide compelling evidence of their full differentiation with formation of single central lumen, for each spheroid is necessary to capture a series of optical images throughout the entire spheroid. This can be accomplished with a Zeiss Axiovert fluorescence microscope by taking a series of five focal sections along the $\mathrm{z}$ axial direction (Fig. 2a) starting from the base and proceeding towards the top of each spheroid double labeled for actin filaments and nuclei. As shown in Fig. 2b, only the optical section taken exactly in the central region of fully differentiated spheroid (focal plane $\mathrm{z}_{3}$ ) reveals the well-defined ring that characterizes these hollow spheroids. By contrast, in those $\mathrm{z}$ optical sections taken through the single layer of cells surrounding the central hollow core (Fig. $2 \mathrm{~b}$, focal planes $\mathrm{z}_{1}, \mathrm{z}_{2}, \mathrm{z}_{4}$ and $\mathrm{z}_{5}$ ), spheroid appears as a round-shape aggregation of cells. Images of panel $2 \mathrm{c}$ confirm that the optical sectioning method is crucial to assess 3D Caco-2 spheroid full differentiation. Indeed, in undifferentiated 3D Caco-2 spheroids without hollow lumen (spheroid in yellow frame) the well-defined ring is never detectable, not even at the central $\mathrm{z}_{3}$ optical section. 

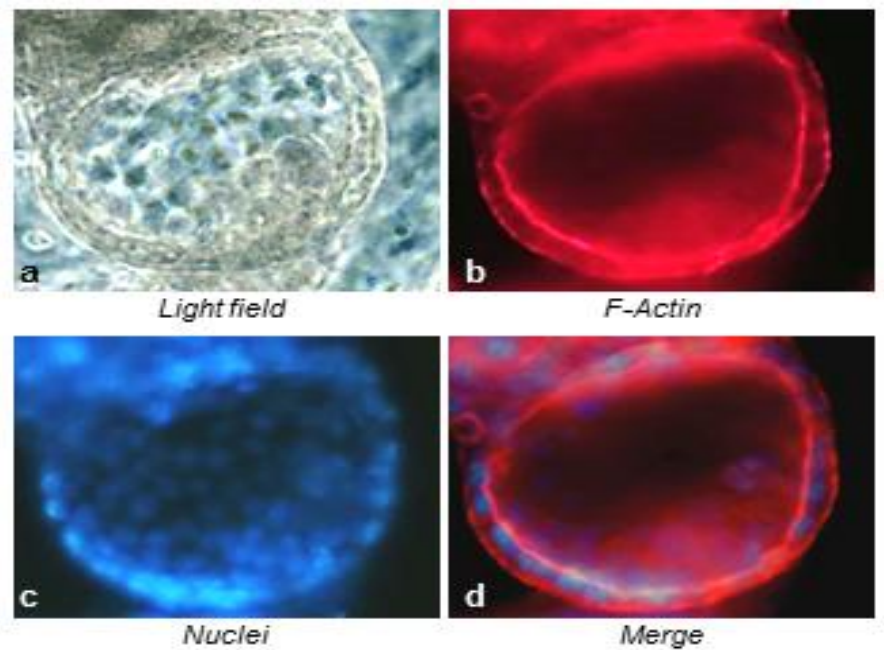

Fig. 1 Representative light (a) and fluorescence microscopic micrographs (b, c, d) of whole fully differentiated 3D Caco-2 spheroids taken with a Zeiss Axiovert inverted light/fluorescence microscopy. Spheroids are double stained with Phalloidin-TRITC to reveal actin organization (b; red) and with Hoechst 33258 to detect nuclei location (c; blue). Merge of the two labelings is shown in (d). Micrographs represent a single microscopic focal plane captured next to the midst of hollow spheroid. As can be seen, the characteristic 3D architecture of hollow Caco-2 spheroids is well evident. In fact, actin filaments have the typical ring appearance (b), and the spatial arrangement of nuclei (c) reveal the single layer of cells that characterize Caco-2 spheroids having a central lumen. Merge between the two stainings (d) is necessary to confirm this spatial arrays. Original magnification: $x 10$ for all micrographs.

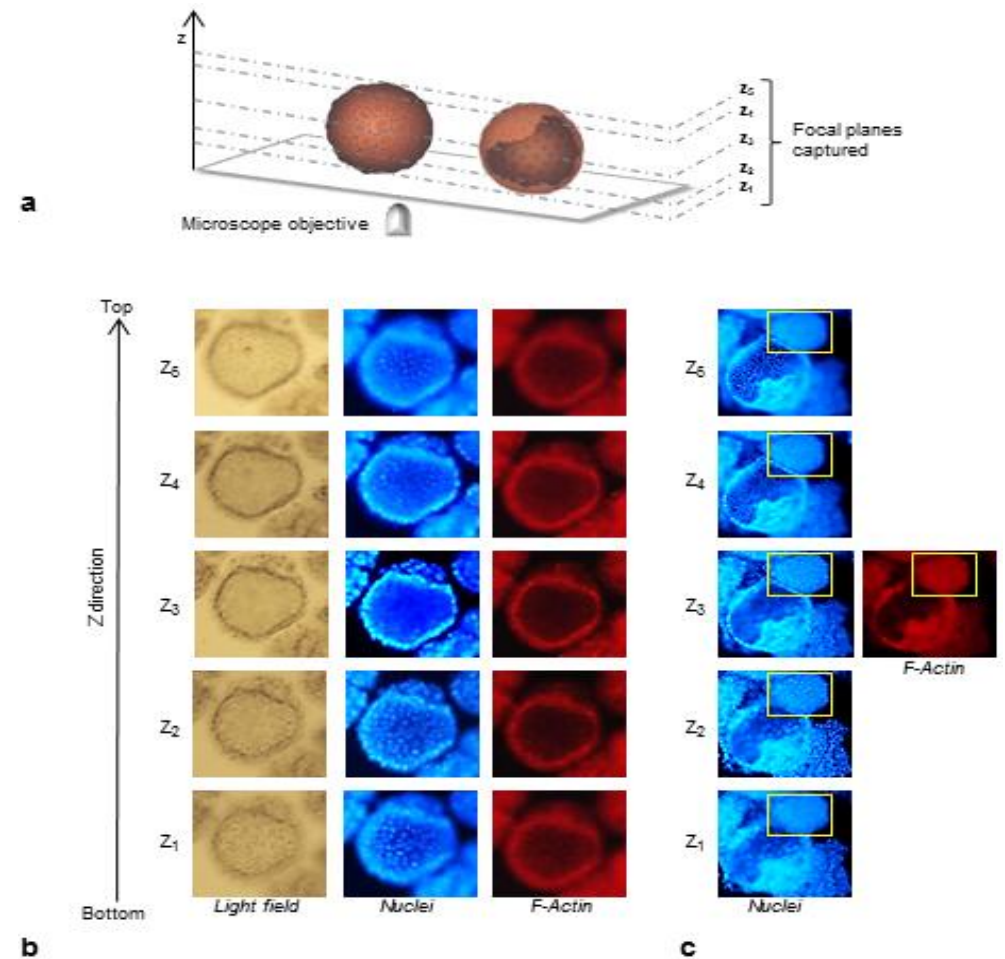

Fig. 2 Fluorescent 3D Caco-2 spheroid differentiation analysis with optical serial sections by using an inverted light/fluorescence microscope. In panel (a) a schematic representation of five serial optical planes captured along the $z$ direction is shown. In panel (b) is the sequence of the five optical sections taken of the same Caco-2 spheroid, double stained with Hoechst to detect nuclei (blue) and Phalloidin-TRITC to reveal actin filaments (red) is shown. The corresponding light images are shown on the left of this panel. In panel (c) the sequence of five optical sections of Caco-2 spheroids, double stained for nuclei and actin filaments (blue and red, respectively) are also shown. Original magnifications: x10 for all micrographs. 


\subsection{Morphological Analyses of 3D Caco-2 Spheroids} Grown in DMEM 2,75 g/l Glucose-5\% FBS

In order to evaluate the influence exerted by DMEM 2,75 g/l glucose-5\% FBS medium on Caco-2 spheroid growth and differentiation, a time-course morphological analysis of spheroids up to 9 days of culture was carried out by light microscopy directly in the tissue plates. As shown in Fig. 3 (a-f), Caco-2 spheroids grown with the DMEM $4.5 \mathrm{~g} / \mathrm{L}$ glucose $-10 \%$ FBS medium are well-formed, appear well-rounded and have well-defined margins. Nevertheless, even at later time of culture, the number of hollow differentiated 3D spheroids is very low. Conversely,
3D Caco-2 spheroids grown with the DMEM 2,75 g/l glucose-5\% FBS medium (Fig. 3, g-n) appear smaller and less in number than those grown with the other protocol. However, a high number of fully differentiated 3D Caco-2 spheroids with a hollow core are formed over the time. These results indicate that the use of the DMEM 2,75 g/l glucose- $5 \%$ FBS medium positively influences 3D Caco-2 spheroid differentiation process. Noteworthy, pairs of Figs. 3 (j-k) and (m-n), each of them showing the same microscopic field captured on consecutive days at the same magnifications, clearly indicate that once 3D differentiated Caco- 2 spheroids are formed their increase
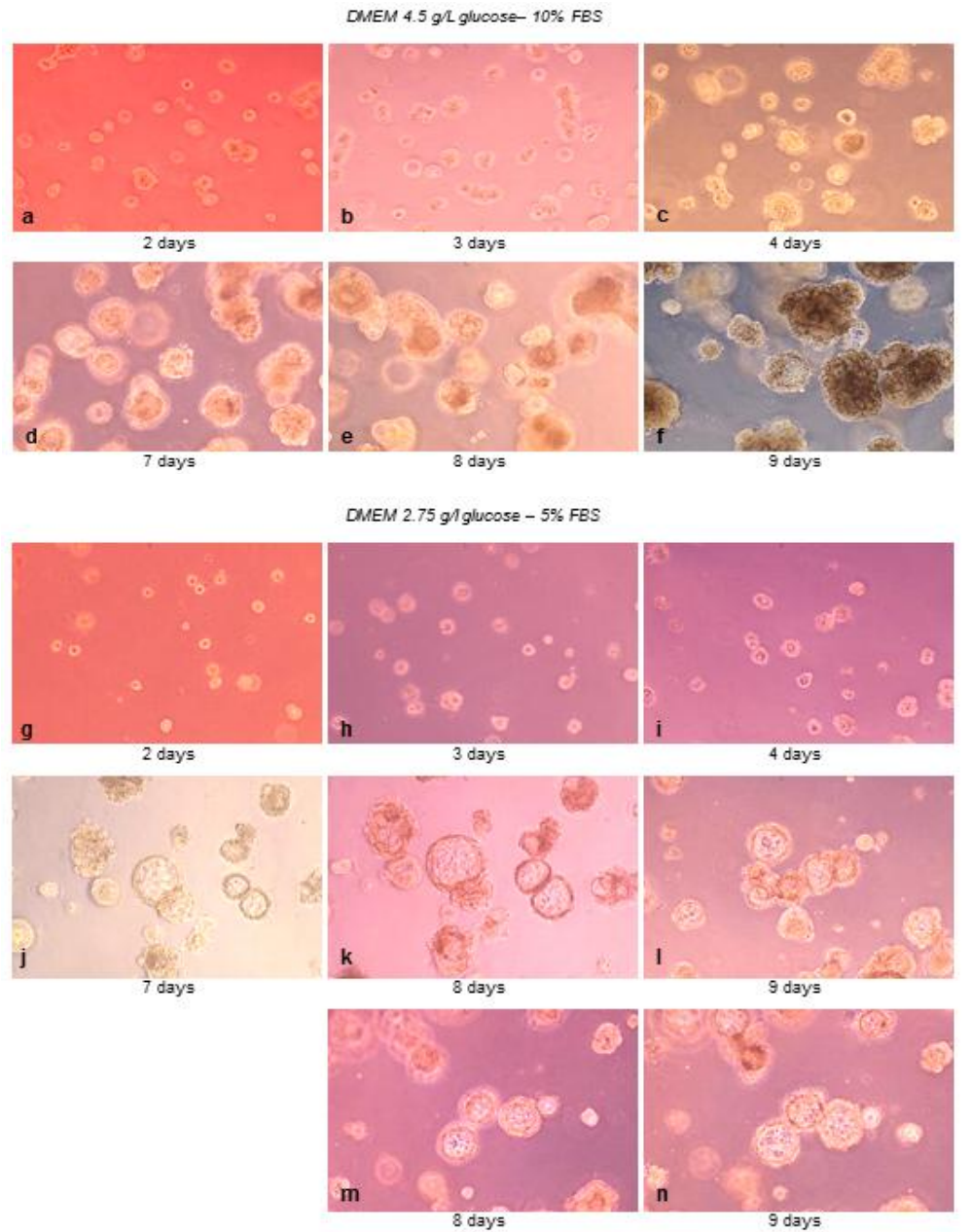

Fig. 3 Representative light microscopic micrographs of 3D Caco-2 spheroids grown by using the DMEM 4.5 g/L glucose-10\% FBS culture method (a-f) and the DMEM 2,75 g/l glucose - 5\% FBS culture system (g-n), up to 9 days of culture. Pairs of micrographs (j-k) and (m-n) show the same microscopic field captured on consecutive days at the same magnifications for each of the pairs. In bracket under each light microscopic image are indicated the culture days at which micrographs have been taken. Original magnification: x10 for all micrographs. 
in size, over the time of growth, is due to the central lumen enlargement, as previously reported [14].

To confirm that the growth protocol 1 favors the differentiation process of 3D Caco-2 spheroids, it was pivotal to ascertain the presence of the ultrastructural parameters characterizing their complete differentiation, i.e., the single layer of cells surrounding a central hollow lumen and the ring of cytoskeletal actin. Consequently, fluorescence double staining for actin filaments and nuclei of Matrigel embedded whole Caco-2 spheroids grown with the two protocols were conducted at 9 days of culture. The entire coverslips of each 3D Caco-2 spheroid sample was observed and photographed with the Zeiss Axiovert microscopy. For each microscopic field four micrographs, i.e., brightfield, Phalloidin-TRITC fluorescence, Hoechst fluorescence and Phalloidin-TRITC/Hoechst fluorescence merge, were captured, at the same focal plane, at low magnification (x 5). As shown in Fig. 4, a higher number of fully
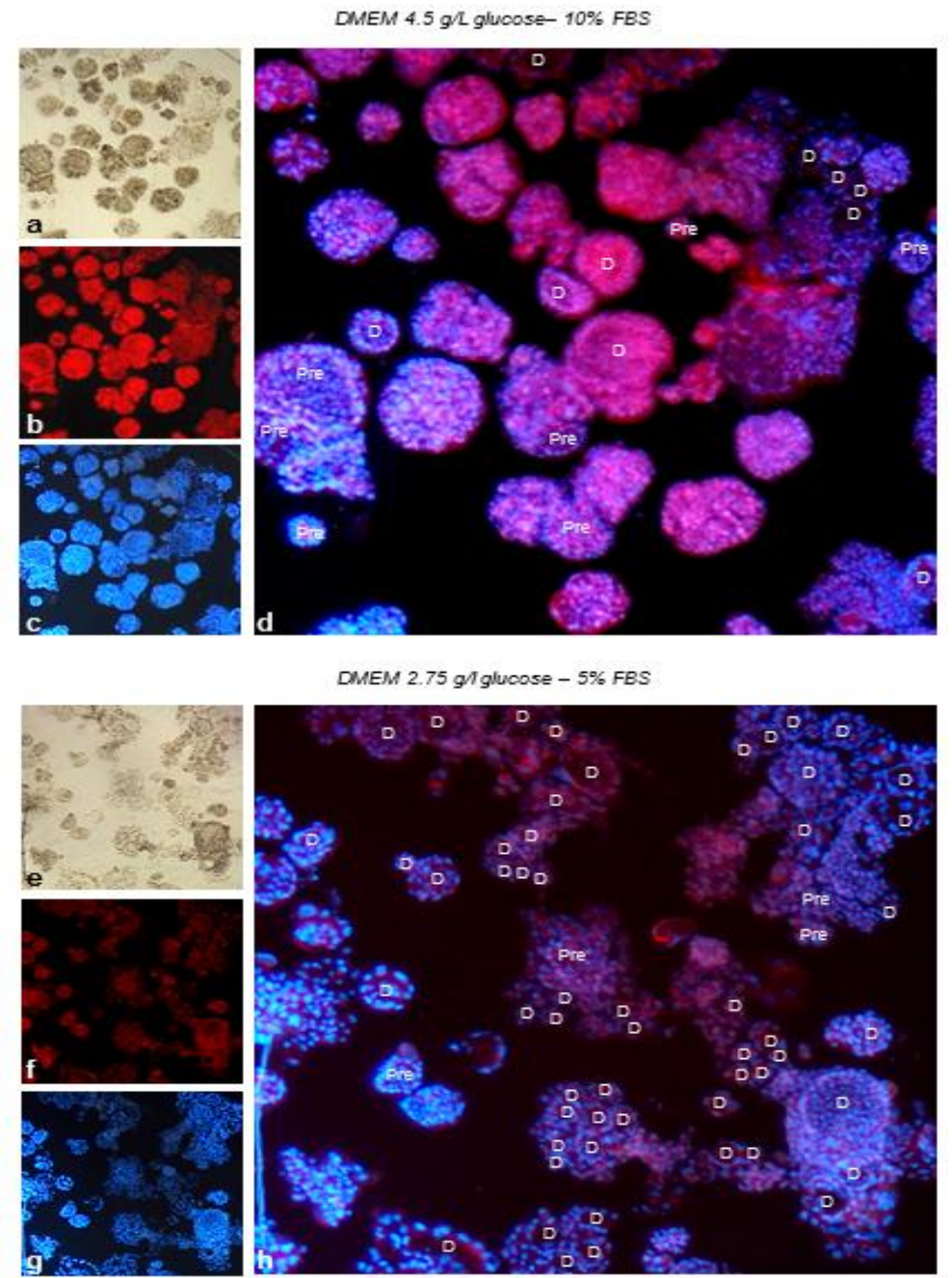

Fig. 4 Representative light and fluorescence microscopic micrographs of 3D Caco-3 spheroids grown by using the DMEM 4.5 g/L glucose-10\% FBS culture method (panel a-d) and the DMEM 2,75 g/l glucose-5\% FBS culture system (panel e-h), at 9 days of culture. The four micrographs of each spheroid samples represent the same microscopic field captured four times as brightfield (a and e), Phalloidin-TRITC fluorescence (red, b and f), Hoechst fluorescence (blue, c and g) and Phalloidin-TRITC/Hoechst fluorescence merge ( $d$ and $h$ ). White " $D$ " letters in merge micrographs indicate fully differentiated 3D Caco-2 spheroids. White "Pre" terms in merge micrographs refer to spheroids with pre-differentiation morphological features in which the actin ring formation and the characteristic spatial location of nuclei have not yet completely occurred. Original magnifications: 55 for all micrographs. 


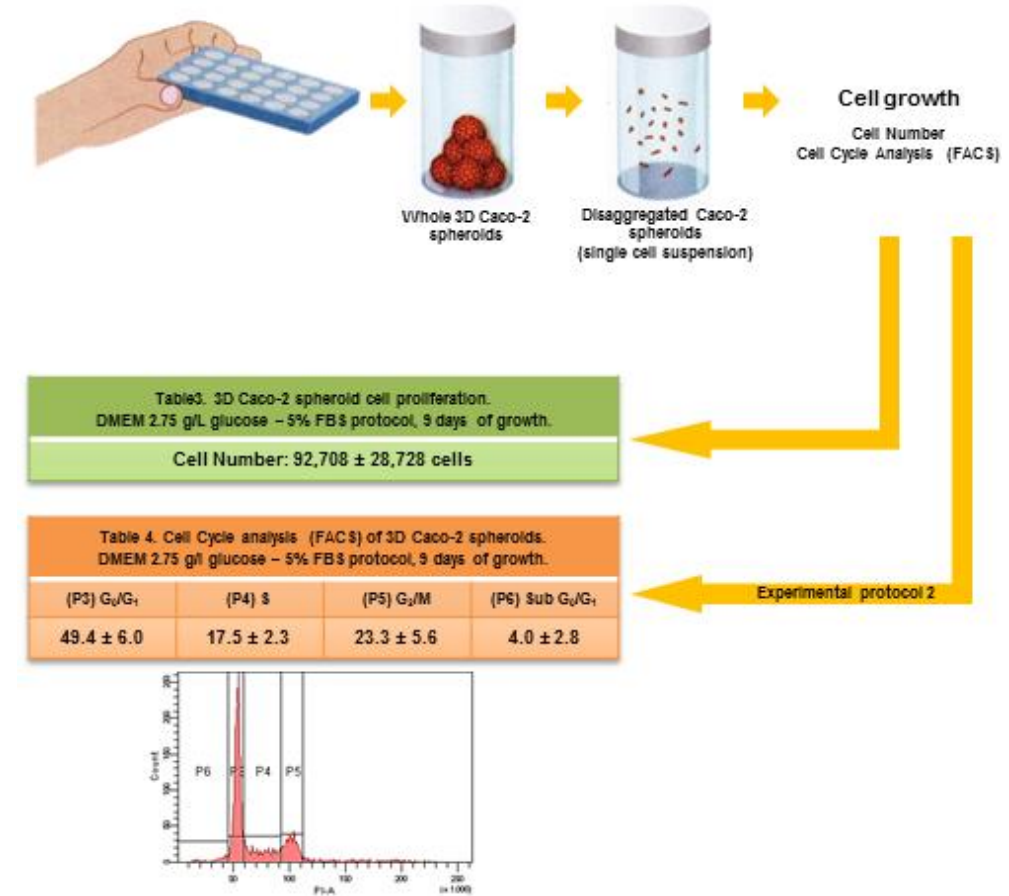

Fig. 5 Schematic diagram of 3D Caco-2 spheroids cell growth analyses. In this schematic representation, experimental protocol 2 section refers to the cell cycle phase investigation by FACS technique, in which a representative FACS histogram of cell cycle phase distribution of disaggregated Caco-2 spheroids at 9 days of growth is shown. P3, P4, P5 and P6 in the FACS histogram correspond to the $G_{0} / G_{1}, S$ and $G_{2} / M$ phases and the sub $G_{0} / G_{1}$ population, respectively.

differentiated 3D hollow Caco-2 spheroids can be observed in the DMEM 2,75 g/l glucose-5\% FBS sample (panel e-h) with respect to the DMEM $4.5 \mathrm{~g} / \mathrm{L}$ glucose-10\% FBS sample (panel a-d). These data confirm the above light microscopy results, namely, that the culture protocol 1 favors 3D Caco-2 differentiation process.

\subsection{Cell Cycle Analysis of Disaggregated Caco-2 Spheroids by FACS}

To evaluate in depth Caco-2 spheroid growth characteristics, proliferation rate and cell cycle phase distribution were assessed.

As shown in the schematic diagram of Figure 5, we first assessed cell proliferation in Caco-2 spheroids isolated from Matrigel cultures and disaggregated as described above. Table 3 in Figure 5 reports the cell numbers of 3D Caco-2 spheroids cultured with DMEM $2,75 \mathrm{~g} / \mathrm{l}$ glucose $-5 \%$ FBS protocol, at 9 days of growth. Cell number is shown as means $\pm \mathrm{SD}$ (standard deviations) of at least 15 separate wells from 6 independent experiments. As shown, these 3D Caco-2 spheroid cultures are characterized by remarkable cellular growth $(92,708 \pm 28,728$ cells $)$ with respect to the number of cells originally seeded (5000 cells).

According to experimental protocol 2, disaggregated Caco-2 spheroid cultures were then analyzed to determine specifically the distribution of the cell cycle phases by FACS. In Figure 5 a representative FACS histogram of the cell cycle phase distribution of disaggregated Caco-2 spheroids at 9 days of growth is shown. As can be seen, the histogram exhibits the typical profile of cells in the three cell cycle phases. The sub $\mathrm{G}_{0} / \mathrm{G}_{1}$ peak, corresponding to apoptotic cells, is also visible. The quantitative analysis of cells in each cell cycle phase is reported in Table 4 of Figure 5. The numbers represent the percentage of cells in $G_{0} / G_{1}, S$ and $G_{2} / M$ phases (means $\pm S D$ ) of at least 11 separate wells from 5 independent experiments. The percentage value of the sub $\mathrm{G}_{0} / \mathrm{G}_{1}$ population, identifying apoptotic cells, is also reported.

These results indicate that the FACS cell cycle 
analysis of disaggregated Caco-2 spheroids respects the key parameters (i.e., $\mathrm{CV}$, and $\mathrm{G}_{2} / \mathrm{G}_{1}$ ratio) [17], described in Methods, used to estimate the quality of a DNA histogram, confirming the adequacy of this protocol to assess their growth features.

\section{Discussion}

In this study, we optimized methods for in vitro culturing 3D Caco-2 spheroids and to assess their growth behavior. The practical and conceptual advantages and benefits of applying these experimental strategies are clearly evident.

Since the quality of 3D Caco-2 spheroid cultures is based on the number of differentiated spheroids with a single lumen [12, 13], our experimental growth condition that optimize the number of fully differentiated spheroids is certainly a technical advance and improvement for all research activities, including ours, that need this type of 3D cell culture model.

The FACS cell cycle analysis of disaggregated 3D Caco-2 spheroids offers diverse advantages. From an experimental point of view, this approach permits the rapid collection of data from a high number of samples grown under identical culture conditions. Conceptually, it allows to be estimated, in relatively short time, specific effects of stressful stimuli on 3D Caco-2 spheroid growth dynamics, besides the visualization of the apoptotic cell death induction.

In addition, it should be emphasized the microscopic analysis of whole well-differentiated 3D Caco-2 spheroid that can be carried out with optical serial sections by using an inverted light/fluorescence microscope. This microscopic approach points out an important practical aspect, namely, that the use of an inverted light/fluorescence microscopy may be a good option allowing researchers to adequately explore 3D Caco-2 spheroid morphology also in laboratories not equipped with confocal microscopy.

The true potential and advantages of using these experimental methods deserve special attention and can be highlighted and summarized in two main aspects. First, all of them are easy to use and time-saving in monitoring 3D Caco-2 spheroid responses to stressful agents. Secondly, the economic savings deriving from their practical application should not be ignored. Thus, the optimized protocols described here represent useful technological approaches to be extended and applied in a systemic manner to the scientific community that use in vitro 3D Caco-2 spheroids to explore intestinal pathophysiology.

Great care should be given to the choice of technologies to investigate specific intestinal epithelial issues. It is hoped that the benefits discussed in this technical note will encourage and stimulate scientists to a more widely use of 3D intestinal spheroid systems to investigate many aspects of intestinal biology, as well as in other research areas.

\section{Funding}

This work was supported by "AIRC (Associazione Italiana Ricerca sul Cancro)" [IG 2013 N. 14185 to SG].

\section{Acknowledgements}

The Authors are grateful to Mr. Cosimo Marino Curianò for his excellent graphic work.

\section{References}

[1] Santini, M. T. et al. 2000. "Apoptosis, Cell Adhesion and the Extracellular Matrix in the Three-Dimensional Growth of Multicellular Tumor Spheroids." Crit. Rev. Oncol. Hematol. 36: 75-87.

[2] Abbot, A. 2003. "Cell Culture: Biology's New Dimension." Nature 424: 870-2.

[3] Debnath, J., and Brugge, J. S. 2005. "Modelling Glandular Epithelial Cancers in Three-Dimensional Cultures." Nat. Rev. Cancer 9: 675-88.

[4] Pampaloni, F. et al. 2007. "The Third Dimension Bridges the Gap between Cell Culture and Live Tissue." Nat. Rev. Mol. Cell Biol. 8: 839-45.

[5] Elliott, N. L., and Yuan, F. 2011. "A Review of Three-Dimensional in vitro Tissue Models for Drug Discovery and Transport Studies." J. Pharm. Sci. 100: 59-74.

[6] Ravi, M. et al. 2009. "Culture Phases, Cytotoxicity and Protein Expressions of Agarose Hydrogel Induced Sp2/0, 


\section{Differentiation Process and to Analyze Their Cell Growth Behavior}

A549, MCF-7 Cell Line 3D Cultures." Cytotechnology (accessed 5 November 2014, epub ahead of print).

[7] Friedrich, J. et al. 2009. "Spheroids-Based Drug Screen: Considerations and Practical Approach." Nat. Protoc. 4: 309-24.

[8] Burdett, E. et al. 2010. "Engineering Tumors: A Tissue Engineering Perspective in Cancer Biology." Tissue Eng. Part B Rev. 16: 351-9.

[9] Ravi, M. et al. 2015. "3D Cell Culture Systems: Advantages and Applications." J. Cell Physiol. 230: 16-26.

[10] Juuti-Uusitalo, K. et al. 2011. "Differential Effects of TNF (TNFSF2) and IFN- $\gamma$ on Intestinal Epithelial Cell Morphogenesis and Barrier Function in Three-Dimensional Culture." PLoS One 6: e22967.

[11] Ludidi, S. et al. 2015. "The Intestinal Barrier in Irritable Bowel Syndrome: Subtype-specific Effects of the Systemic Compartment in an in vitro Model." PLoS One 10: e0123498.

[12] Elamin, E. et al. 2012. "Effects of Ethanol and Acetaldehyde on Tight Junction Integrity: in vitro Study in a Three Dimensional Intestinal Epithelial Cell Culture Model." PLoS One 7: e35008.

[13] Elamin, E. et al. 2013. "Fatty Acid Ethyl Esters Induce Intestinal Epithelial Barrier Dysfunction via a Reactive Oxygen Species-Dependent Mechanism in a Three-Dimensional Cell Culture Model." PLoS One 8: e58561.

[14] Lechler, T. 2008. "Limiting Lumens: A New Role for Cdc42." J. Cell Biol. 183: 575-7.

[15] Jaffe, A. B. et al. 2008. "Cdc42 Controls Spindle Orientation to Position the Apical Surface during Epithelial Morphogenesis." J. Cell. Biol. 183: 625-33.

[16] Debnath, J. et al. 2003. "Morphogenesis and Oncogenesis of MCF-10A Mammary Epithelial Acini Grown in Three-Dimensional Basement Membrane Cultures." Methods 30: 256-68.

[17] Ormerod, M. G. 2003. DNA Analysis. In: Ormerod MG. ed. Flow Cytometry. A Practical Approach. Oxford: Oxford University Press, 3rd edition, 83-97. 